\title{
Determining Motions and Birthplaces of Pulsars through VLBI Astrometry
}

\author{
T. J. Galama, J. Van Paradijs ${ }^{1}$, \& E. P. J. van den Heuvel \\ Astronomical Institute 'Anton Pannekoek'/ CHEAF, University of Amsterdam, \\ Amsterdam, The Netherlands
}

A. G. de Bruyn ${ }^{2}$, R. M. Campbell, \& R. C. Vermeulen

NFRA, Radio Observatory, Dwingeloo, The Netherlands

J.-F. Lestrade

Observatoire de Paris, Paris, France

F. Verbunt

Astronomical Institute Utrecht, Utrecht, The Netherlands

R. T. Schilizzi

Joint Institute for VLBI in Europe (JIVE), Dwingeloo, The Netherlands

\begin{abstract}
We present first results of global VLBI astrometric pulsar parallax and proper motion measurements (phase-reference). The aim is to obtain information on pulsar motions and pulsar birthplaces. Proper motions could provide answers to questions like: How large are pulsar velocities at birth? How are these velocities produced and what is the final galactic pulsar distribution? Identification of birthplaces (with, e.g., an OB-association) provides information on the pulsar progenitor population (the fraction of pulsars born in binaries; the mass range of the progenitors etc.). We have a first epoch on three pulsars, selected on the basis of age (young $<3 \mathrm{Myr}$ ), flux density (relatively strong) and presence in the solar neighborhood ( $d<$ $3 \mathrm{kpc}$ ). Gating increases the SNR by typically a factor of 5 .
\end{abstract}

\section{Introduction}

It is generally believed that most, if not all, pulsars (i.e. neutron stars) are formed in the explosion of a massive population I star. Yet, very little direct evidence to support this belief is available. The origin of pulsars is intimately linked to a number of important questions of stellar evolution. Relevant questions that can be posed are: How large are pulsar velocities at birth? What is (are) the pulsar progenitor population(s)? Are there pulsars born at high distances from the galactic plane? What is the mass range of pulsar progenitors? Which fraction of pulsars was born as a member of a binary? Accurate determinations of pulsar velocities and distances are the key to answering many of these questions.

If we can accurately measure the transverse velocity of a young pulsar (where young means less than a few Myr) it should be possible to trace back its orbit in (the sufficiently well-known) galactic gravitational field and directly infer its birth place. Identification with an existing OB-association would be a very significant discovery since it would then permit accurate calibration of the pulsar "kinematic" age and the mass of its progenitor, amongst other parame-

\footnotetext{
${ }^{1}$ Also at Physics Department, University of Alabama, Alabama, USA

${ }^{2}$ Also at Kapteyn Astronomical Institute, Groningen, The Netherlands
} 
ters. It would also give us a lower limit to the mass of stars which end their life as a black hole rather than a neutron star.

We expect that young pulsars and associations can in many cases be matched convincingly, if they are indeed associated, on the basis of the projected velocity only. Computer simulations (J-W. Hartmann, private communication) show this expectation to be borne out. An unambiguous "match" will also allow us to test the usual assumption that $0.5 P /(d P / d t))$, the "spin-down age", is an accurate measure of the true age of the pulsar. The effect of parallax is non-negligible if we can reach sub-mas accuracies in (relative) position. That sub-mas astrometry is possible has also been shown in the recent work of Campbell et al. (1996).

\section{Results from the First Epoch (May 1995)}

We obtained 5-6 hours of coverage on three pulsars using a 8 station network (BWS in Europe, GHIVY in US). Using 20 milliperiod gates, a SNR gain of about a factor 4-5 was obtained on all three pulsars. A brief description of these results follows: For PSR B2020+515.5 hours of data were obtained with cycles of 10 minutes (cycling over the PSR +3 reference sources) and, for part of the time, 7.5 minutes (PSR +2 refs). Using more than one reference source the errors in the astrometry and the effects of long-range ionospheric phase-delay gradients can be investigated, and hopefully removed. All reference sources (at angular separations from the pulsar ranging from 0.3-1.5 degrees) were detected with a SNR $>20$ in 70-90 seconds of net integration on all baselines. PSR B2020 +51 was unusually bright during most of the 5 hours we observed it, with a SNR ranging from 10 to more than 1000 . For PSR B1508+55 data were obtained with a cycle time of $7.5 \mathrm{~min}$ using 2 reference sources. Both the pulsar and the 2 reference sources were detected for $(>90 \%)$ of the time. In addition to rapid intensity fluctuations, our bandwidth $(7 \times 4 \mathrm{MHz})$ was wide enough to also observe large $(>10$ !) amplitude scintillation across the 7 IF channels. In principle a further SNR-gain of a factor $>2$ can be obtained by using only those IFs where the pulsar was bright. Methods to implement this "frequency gating" are under investigation. This pulsar is extraordinary in that the proper motion gives this pulsar a very high space velocity $(900 \mathrm{~km} / \mathrm{sec} !)$, assuming its dispersion measure distance. A derivation of the parallax (or an upper bound) should enable us to confirm this unusually large space velocity. PSR B0450+55 was much weaker than expected and was detected with SNR $>6$ for only part of the time (but most of the time on baselines to Effelsberg). The reference sources were detected with good SNR. Using proven methods (e.g., Alef 1987, Lestrade et al. 1992) we can recover the pulsar signal.

\section{References}

Alef, W. 1987, Ph.D. Thesis, Bonn.

Campbell, R. M. et al. 1996. ApJ, 461, L95-98.

Hartman, J.-W. et al. 1997. $A \& A, 461,698-712$.

Lestrade, J. F. et al. 1992. A $\& A, \mathbf{2 5 8}, \mathrm{L112-115.}$

Lorimer, D. R. et al. 1995. MNRAS, 273, 411-421. 